\title{
Correction to: Two-Stage Method for Segmentation of the Myocardial Scars and Edema on Multi-sequence Cardiac Magnetic Resonance
}

Yanfei Liu, Maodan Zhang, Qi Zhan, Dongdong Gu, and Guocai Liu

\section{Correction to:}

Chapter "Two-Stage Method for Segmentation of the Myocardial Scars and Edema on Multi-sequence Cardiac Magnetic Resonance" in: X. Zhuang and L. Li (Eds.): Myocardial Pathology Segmentation Combining Multi-Sequence Cardiac Magnetic Resonance Images, LNCS 12554, https://doi.org/10.1007/978-3-030-65651-5_3

The original version of this chapter was revised. The institute Shanghai United Imaging Intelligence, Co., Ltd., Shanghai, China of the author Dongdong Gu has been removed and the acknowledgement has been changed to "This work was supported by the National Natural Science Foundation of China (61671204)." 